\title{
Natural immunity to influenza $A$ and $B$ among Saudi blood donors in Al Madinah Al Munawarah, Saudi Arabia
}

Waleed H. Mahallawi, PhD, Nadir A. Ibrahim, PhD, Khalid S. Alahmadi, BSc, Abdullah K. Al-Harbi, BSc, Mohammed A. Almughthawi, BSc, Omar A. Alhazmi, PhD, Fayez H. Alsebli, MSc, Omar F. Khabour, PhD.

\begin{abstract}
الأهداف: معرفة مدى انتشار الأجسام المضادة في مصل المتبرعينٍ

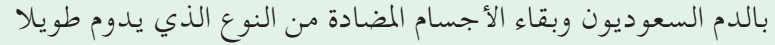

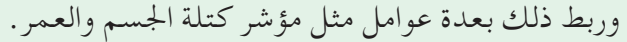

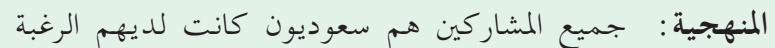

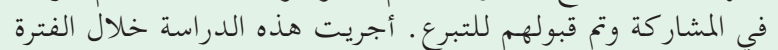

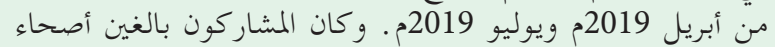

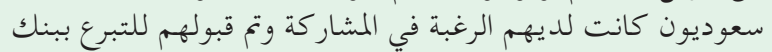

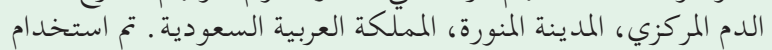

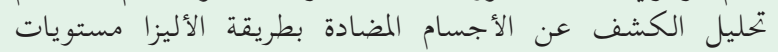

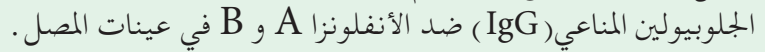

النتائج : أظهرت النتائج أن الإنفلونزا من النوع أ كانت انتشارها بين

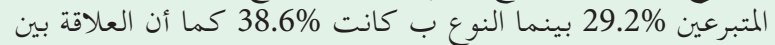

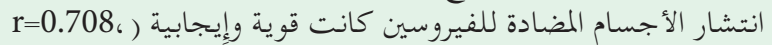

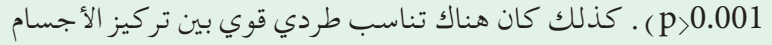

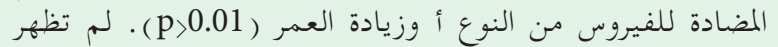

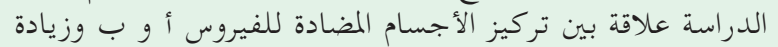

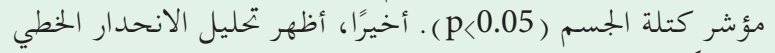

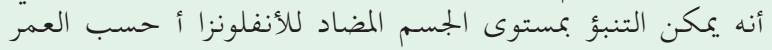

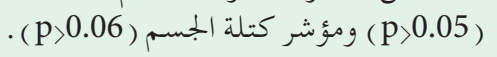

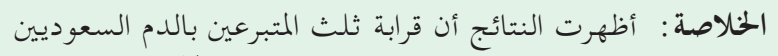

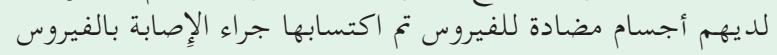

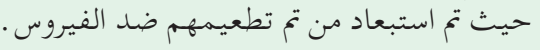

Objectives: To investigate the seroprevalence of influenza viruses (A and $\mathrm{B}$ ) among blood donors in Kingdom of Saudi Arabia.

Methods: The present investigation was conducted between April 2019 and July 2019. Participants were healthy adults recruited from the central blood bank $\mathrm{Al}$ Madinah Al Munawarah, Kingdom of Saudi Arabia. Immunoglobulin $\mathrm{G}$ ( $\mathrm{IgG}$ ) levels against influenza A and $\mathrm{B}$ were measured in serum samples using ELISA.
Results: The results showed that $29.2 \%$ of the sample had significant concentrations of influenza A IgG antibody, whereas $38.6 \%$ had significant concentrations of influenza B IgG antibody. A strong correlation was found between the levels of influenza $A$ and influenza B antibodies $(r=0.708, p<0.001)$. The number of individuals identified as negative for influenza A IgG antibody increased with age $(p<0.01)$. In addition, no correlations were identified between influenza A IgG and influenza B IgG and body mass index (BMI), $(p>0.05)$. Finally, linear regression analysis showed that the level of influenza $A$ antibody can be predicted by age $(p<0.05)$ and body mass index (BMI) $(p<0.05)$.

Conclusion: Approximately one-third of Saudi Arabian adults presented significant levels of influenza $A$ and $B$ antibodies in our study. Demographic factors, including age and BMI, might contribute to influenza A antibody levels.

Keywords: influenza A, influenza B, seroprevalence, IgG, Al Madinah Al Munawarah

Saudi Med J 2020; Vol. 41 (12): 1301-1307 doi: 10.15537/smj.2020.12.05582

From the Department of Medical Laboratory Technology (Mahallawi, Ibrahim, Alahmadi, Al-Harbi, Almughthawi), College of Applied Medical Sciences, Taibah University, from the General Directorate of Laboratories and Blood Banks (Alhazmi, Alsehli), Ministry of Health, Al Madinah Al Munawarah, Kingdom of Saudi Arabia and from the Department of Medical Laboratory Sciences (Khabour), Faculty of Applied Medical Sciences, Jordan University of Science and Technology, Irbid, Jordan.

Received 28th July 2020. Accepted 21st November 2020.

Addresscorrespondence andreprint request to: Dr. Waleed H. Mahallawi, Department of Medical Laboratory Technology, College of Applied Medical Sciences, Taibah University, Al Madinah Al Munawarah, Kingdom of Saudi Arabia.E-mail: wmahallawi@taibahu.edu.sa ORCID ID: https://orcid.org/0000-0001-6977-9006 
Tnfluenza is a highly transmittable, acute viral infection of the respiratory tract. ${ }^{1}$ Influenza belongs to the family Orthomyxoviridae, and 4 types of influenza have been identified, including influenza $A$ and $B$, which are enveloped ribonucleic viruses. ${ }^{2,3}$ The global burden of influenza is high. ${ }^{4,5}$ Approximately, 100,000 individuals die each year from direct influenza virus infections of the lower respiratory tract. ${ }^{4}$ In addition, more than one-quarter million individuals die from influenza-related complications globally. ${ }^{6,7} \mathrm{~A}$ recent review highlighted the extra-pulmonary complications (damage to organs other than the lungs) associated with influenza infections that can increase the healthcare burden associated with influenza infections. ${ }^{8}$ Individuals with underlying illnesses, such as respiratory and cardiovascular diseases, reduced immunity, and asthma, are at high risk of developing severe influenza symptoms, which may result in hospital admission. The implementation of influenza vaccine programs might limit the spread of the disease to susceptible individuals and decrease the overall burden of the disease. ${ }^{9}$ The usefulness of vaccination programs depends largely on the selection of appropriate vaccines that target those influenza strains that are common to the country in question. Therefore, determining the seroprevalence of the population is important for guiding the selection of influenza vaccine strains.

The Ministry of Health (MOH), Kingdom of Saudi Arabia (KSA) has established a free influenza vaccination that is available at all primary health care facilities. However, limited studies were performed to investigate the effectiveness of this vaccine in this country. For example, in a small sample-size study conducted in $\mathrm{Al}$ Madinah Al Munawarah, KSA, the influenza vaccine was shown to be effective for initiating humoral immune responses in recipient subjects. ${ }^{10}$ Additional national studies remain necessary to examine the effectiveness and persistence of anti-influenza antibodies among vaccinated individuals. In general, natural influenza virus infections produce wide-ranging and long-lasting immunity. In contrast, the immune response that develops in response to vaccination is narrow and shortlived. ${ }^{11,12}$

In the current study, healthy blood donors were examined to investigate the current seroprevalence of influenza A and B Immunoglobulin G ( $\operatorname{IgG})$

Disclosure. Authors have no conflict of interests, and the work was not supported or funded by any drug company. antibodies in KSA. Blood donors have also been used in similar previous studies performed in Australia and in Germany. ${ }^{13,14}$

Other factors, such as age and obesity, have been shown to affect the humoral immune response. ${ }^{15,16}$ For example, a recent study showed that age was a valid predictor of the humoral immune response to pathogens and vaccines. Reduced and delayed immune responses to influenza viruses have also been reported in obese individuals. ${ }^{15,16}$ In addition, a study conducted on a trivalent influenza vaccine showed a greater decline in the antibody titer in obese individuals compared with that in normal-weight individuals when examined 12 months after vaccination. ${ }^{17}$

The study aim to investigate the seroprevalence of influenza A and B IgG antibodies in Al Madinah Al Munawarah, KSA. In addition, the results were compared based on various demographic parameters, including age and body mass index (BMI).

Methods. This study was conducted using a crosssectional design and was conducted between April 2019 and July 2019. All participants $(\mathrm{N}=360)$ were healthy adults ( $>18$ years old), recruited among blood donors at the central blood bank (CBB), Al Madinah Al Munawarah, KSA. On average, approximately 90 individuals donate blood to the $\mathrm{CBB}$ each day. During the recruitment period, every $5^{\text {th }}$ subject from the list of donors was invited to participate in the study. Subjects with human immunodeficiency virus (HIV), hepatitis B virus (HBV), human T-cell lymphotropic virus (HTLV), hepatitis $\mathrm{C}$ virus (HCV), syphilis, and malaria were excluded from the study. Any subject who has ever received a flu vaccine was also excluded $(n=13)$. The Human Research Ethics Committee of the College of Applied Medical Sciences at Taibah University, KSA, approved the study procedures (IRB No: SREC/ AMS 2019/62/CLD). Written informed consent was obtained from all subjects after a full explanation of the study objectives was provided. The sample size was calculated using $G$ power, version 3, and at least 240 participants were estimated to be necessary to achieve a power of 0.8 , an alpha of 0.05 , and moderate effects.

Blood samples were obtained in plain tubes. After coagulation was completed, the samples were immediately centrifuged at $4,000 \times \mathrm{g}$ for 5 minutes. The serum was collected, aliquoted into $1.5 \mathrm{ml}$ tubes, and immediately stored at $-20^{\circ} \mathrm{C}$ until used for enzymelinked immunosorbent assay (ELISA) testing.

Demographic data, including age, gender, nationality, and influenza vaccination status, were obtained using a structured questionnaire. Weight was 
obtained using a digital body weight scale, whereas height was measured using a wall-based body height chart. Body mass index was calculated from weight and height, as previously described. ${ }^{18}$

Indirect ELISA was used to measure influenza $A$ and influenza B IgG antibody levels in the samples (Vircell, Granada, Spain, catalog number L-G/ M1007-EN-02 and L-G/M1008-EN-02), according to the manufacturer's instructions. The ELISA plates were quantified using an EL x 808 Absorbance Reader (BioTek, USA) at 450/620 nm. When the level of IgG was below the cut-off value, the subject was considered negative. However, when the IgG level was above the cut-off value, the subject was considered positive. Equivocal results were also interpreted, according to the kit instructions. ${ }^{19}$

Statistical analysis. The Statistical Package for Social Sciences, version 22 (IBM Corp, Armonk, NY, USA) was used to perform data analysis. Pearson's correlation coefficient and linear regression analyses were conducted to analyze the relationships between IgG data and demographic variables. A $p$-value $\leq 0.05$ was considered significant.

Results. A total of 360 individuals volunteered to participate in this study from the $\mathrm{Al}$ Madinah Al Munawarah region, KSA. The response rate was $62 \%$. The demographic characteristics of the sample population are presented in Table 1 . The mean age was $32.5 \pm 10.2$ years, and the mean BMI was 28.67 \pm 5.9 (range 14.9-49.2). Among all participants, approximately $96 \%(\mathrm{n}=345)$ were nonsmokers. All participants were healthy blood donors and did not suffer from any chronic or inherited diseases.

The ELISA assay was used to measure influenza A and B IgG antibody concentrations for all 360 participants. Figure 1 shows the distribution of participants according to the measured levels. Among the sample population, $29.2 \%$ of individuals $(n=105)$ had significant concentrations of influenza A antibody, whereas $61.4 \%$ participants $(n=221)$ were identified as negative and $9.4 \%(n=34)$ were identified as equivocal. Additionally, $38.6 \%$ of participants $(n=139)$ had significant concentrations of influenza B antibody, whereas $56.1 \%(n=202)$ participants were found to be negative and $10.6 \%(n=39)$ of participants were found to equivocal.

Among the sample population, only 25\% ( $\mathrm{n}=90)$ presented significant levels of both influenza A and B IgG antibodies simultaneously. Furthermore, a strong correlation was found between the levels of influenza A and influenza B IgG antibodies $(r=0.708, p<0.001)$,
Table 1 - Demographic data of participants.

\begin{tabular}{lc}
\hline Parameter & $\mathbf{n}(\%)$ \\
\hline Mean age (years) & $32.5 \pm 10.2$ \\
Age range & $18-66$ \\
Mean BMI & $28.67 \pm 5.9$ \\
BMI range & $14.9-49.2$ \\
BMI categories & \\
$<18.5$ & $5(1.3)$ \\
$18.5-24.9$ & $101(28.1)$ \\
$25-29.9$ & $125(34.7)$ \\
$30-39.9$ & $112(31.1)$ \\
$>40$ & $17(4.7)$ \\
Gender & \\
Male & $360(100)$ \\
Female & $0(0)$ \\
Tobacco use & \\
Yes & $15(4.2)$ \\
No & $345(95.8)$ \\
\hline
\end{tabular}

BMI: body mass index

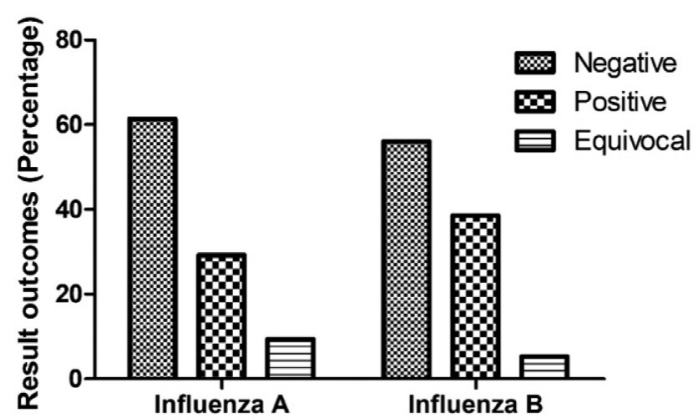

Figure 1 - Distribution of participants according to influenza $\mathrm{A}$ and influenza $B$ antibodies. Influenza $A$ and influenza $B$ antibodies were measured using enzyme-linked immunosorbent assay (ELISA) of serum samples. Values less than $9 \mathrm{IU} / \mu \mathrm{L}$ were considered negative. Values between 9-11 IU/ $/ \mathrm{L}$ were considered equivocal. Values more than $11 \mathrm{IU} / \mu \mathrm{L}$ were considered positive.

using Pearson's correlation coefficient analysis (Figure 2).

To examine the impacts of age and BMI on influenza A and B antibody levels, participants were stratified into various subgroups (Figures $3 \& 4$ ). A significant negative correlation was found between influenza $A$ antibody levels and participant age $(\mathrm{r}=-0.138,95 \%$ confidence interval (CI): [-0.238- -0.035], $p=0.009)$. No significant correlation was detected between age and influenza B antibody levels $(\mathrm{r}=-0.028,95 \% \mathrm{CI}$ : [-0.1316-0.07498], $p=0.588$ ), (Figure 3).

Figure 4 shows the relationships between BMI and antibody levels. No significant correlations were 


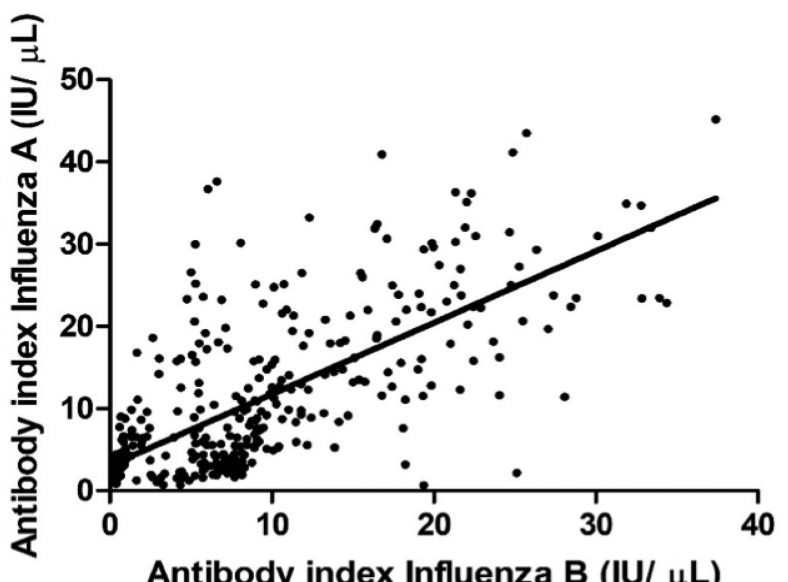

Figure 2 - Correlations between the levels of influenza A and influenza B antibodies. Values are expressed in IU/ $\mu \mathrm{L}$. A strong correlation was found between the level of influenza $\mathrm{A}$ antibody and the level of influenza B antibody $\left(r^{2}=0.503, p<0.001, N=360\right)$.

A)

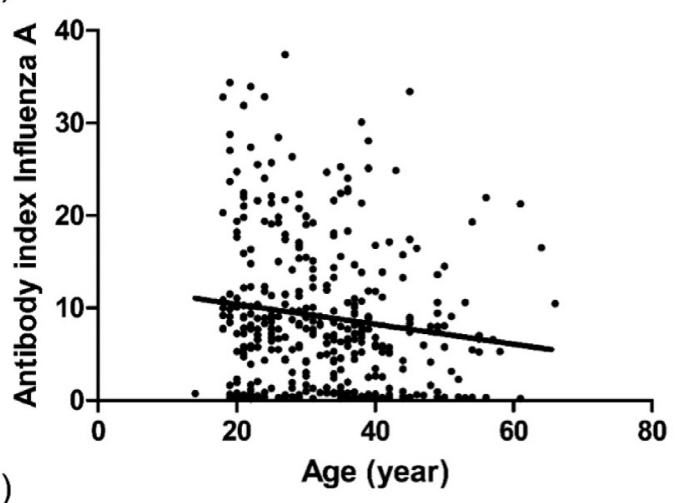

B)

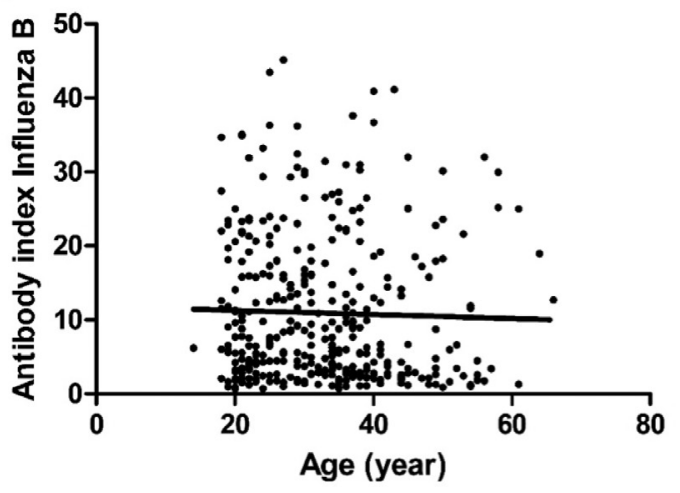

Figure 3 - Correlations between the levels of influenza A and influenza B antibody and age. A) A significant correlation between influenza A antibody levels and age was found $(r=-0.138$, $p<0.01)$. B) No correlation between influenza B antibody levels and age was found $(r=-0.028, p>0.05)$. detected between BMI and either influenza A antibody levels ( $\mathrm{r}=-0.055,95 \%$ CI: [-0.1584-0.04777], $p=0.290$ ), or influenza B antibody levels $(\mathrm{r}=0.0186,95 \% \mathrm{CI}$ : [-0.1393-0.2004], $p=0.724)$, (Figure 4).

Linear regression analysis $(\mathrm{R}=0.1763, \mathrm{R} 2=0.0314$, adjusted $\mathrm{R} 2=0.026$ ) showed that the level of influenza $\mathrm{A}$ antibody can be predicted by age $(\mathrm{B}=-0.112, p=0.018$, 95\% CI: $[-0.205$ to -0.020$])$ and BMI $(B=-0.104$, $p=0.046,95 \%$ CI: [-0.206 to -0.002$])$. However, linear regression analysis showed no association between influenza $\mathrm{B}$ antibody levels and either age $(B=-0.040$, $p=0.43,95 \% \mathrm{CI}:[-0.141-0.060])$ or BMI $(\mathrm{B}=-0.046$, $p=0.41,95 \%$ CI: $[-0.157-0.064])$.

Discussion. In this study, the seroprevalences of influenza $A$ and $B \operatorname{IgG}$ antibodies were examined in Al Madinah Al Munawarah, KSA. The results showed that $29.2 \%$ of the studied population had significant

A)
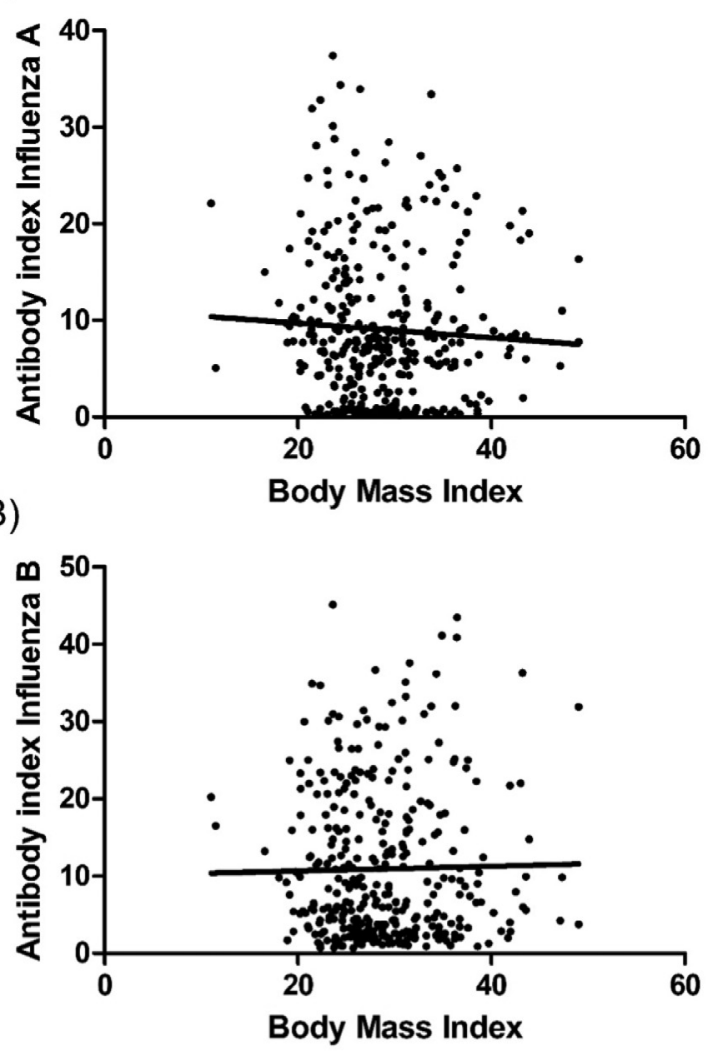

Figure 4 - Correlations between the levels of influenza $\mathrm{A}$ and influenza $\mathrm{B}$ antibody and BMI. A) No correlation between influenza A antibody levels and BMI was found $(r=-0.055, p>0.05)$. B) No correlation between influenza B antibody levels and BMI was found $(\mathrm{r}=0.0186, p>0.05)$. 
concentrations of influenza A IgG antibody, whereas $38.6 \%$ had significant concentrations of influenza B IgG antibody. In addition, a strong correlation was found between the levels of influenza A IgG and influenza B IgG antibodies, and a strong negative correlation was found between influenza A IgG antibody levels and age.

According to the present findings, approximately $29.2 \%$ of the sample population were positive for influenza A IgG antibody. Because the study participants were restricted to those who have never received an influenza vaccine, these significant levels of influenza A IgG antibody are likely due to humoral immune responses due to influenza infections during the individuals' life times. The reported influenza A IgG-positive frequency in this study was similar to that from another study performed in the Middle East, which reported a prevalence of $24 \%$ for Influenza A (H3N2) IgG. ${ }^{20}$ In a recent study performed in KSA and based on the information obtained from the influenza A (H1N1) surveillance project, which was carried out from 2010-2016, a seasonal trend was observed, with a fatality rate of approximately $3.6 \% .{ }^{21}$ When examining influenza B, approximately $38.6 \%$ of our sample population were positive for influenza B IgG antibody. A similar percentage was reported by a study conducted in Riyadh, KSA in 2016, with a reported prevalence of $35 \%$ for influenza B IgG-positive individuals. However, the sample size examined in Riyadh study was relatively small $(n=100)$ and the nationality of the participants was not disclosed. ${ }^{22}$ Infection with influenza $\mathrm{B}$ is frequently misinterpreted as a more minor disease than infection with influenza A. However, several studies have verified that influenza B can also cause epidemics that result in a significant proportion of people requiring hospitalization, similar to those driven by influenza A. ${ }^{23,24}$ Typically, B-Victoria infects naive children/adolescents, whereas B-Yamagata infects both children and adults. ${ }^{25-27}$

The influenza vaccine is seasonal and is recommended to be administered in KSA from September-March, which corresponds to the seasonality of Influenza in KSA. Influenza B virus has been shown to contribute considerably to winter respiratory disease, causing seasonal epidemics every 2 to 4 years. Influenza A epidemics have been reported to peak approximately 2 to 3 weeks earlier than influenza B epidemics in most Gulf states. ${ }^{20}$ In the present study, samples were collected after the winter season, slightly after exposureto the viruses would be expected. Approximately one-quarter of participants were IgG-positive for both influenza strains A and B. Many factors are associated with influenza infections, such as exposure, transmission pressure, which is influenced by crowding or population density, seasonality, and duration. Thus, the fraction of the Saudi Arabian population that were IgG-positive for both influenza strains appears to be more susceptible to influenza virus infection compared with the rest of the sample population. In general, human immunity reproduces and is significantly prejudiced via former influenza infections or vaccinations. ${ }^{28,29}$ More studies remain necessary to investigate these findings.

The association between influenza A IgG levels and the age of participants agrees with the findings of other studies, which showed an age-dependent immune response. $^{30,31}$ The acquisition of higher antibody titrations among young participants is most likely due to recurrent viral exposure viruses and the presence of a strong immunological memory response compared with older individuals. However, among children, an inverse relationship between age and IgG influenza A and B levels has been reported in the Netherlands. Therefore, more studies of influenza viruses in KSA are necessary to examine the patterns of infection and epidemiology and to uncover the mechanisms that under lie the observed association between age and antibody titration.

The present study did not find any correlation between BMI and either influenza A or B IgG levels. However, linear regression analysis indicated that influenza A IgG levels were predicted by BMI. This result agreed with those reported by previous studies, in which BMI correlated with the immune response to influenza and other bacterial respiratory infections. ${ }^{15-17,32}$ In addition, epidemiological data have suggested a relationship between body weight, the risk of infection, and the outcomes of infectious diseases. ${ }^{32}$

The majority of studies conducted in KSA examining the spread of respiratory infections (including influenza) have been performed among Hajj and Umrah populations. ${ }^{33-36}$ The collection of comprehensive data regarding the prevalence of influenza viruses in KSA remains necessary. Thus, systematic national laboratory surveillance is strongly recommended to provide a clear picture of the spread of viral infections throughout the country. The findings presented in this study might provide feedback to the healthcare stake holders to explore the dynamics of viral transmission throughout the country and identify prevention measures to minimize the spread of influenza and, consequently, lower the infection burden on health sectors. Moreover, implementing a national vaccination campaign would be a useful prevention method to improve the acquired immunity against influenza viruses. 
Study limitations. Among the study limitations is the stratification of the sample into different age and BMI categories. Therefore, the confirmation of the present findings in a larger study sample is strongly recommended. In addition, the present study was conducted on blood donors in $\mathrm{Al}$ Madinah $\mathrm{Al}$ Munawarah, KSA, which may induce population biases. Therefore, expanding the study to include other regions in KSA, with a better representation of the overall population, is also recommended for future investigations.

In conclusion, the present study reported the seroprevalences of influenza A and B IgG antibodies in a population in $\mathrm{Al}$ Madinah $\mathrm{Al}$ Munawarah, KSA. Approximately $29.2 \%$ of the sample population had significant concentrations of influenza A IgG antibodies and $38.6 \%$ of influenza B IgG antibodies. In addition, in one-quarter of the sample, significant concentrations of both influenza A and B IgG antibodies were found. Factors such as age and BMI might contribute to Influenza A IgG levels. These results can provide feedback to healthcare providers in $\mathrm{Al}$ Madinah $\mathrm{Al}$ Munawarah, KSA, although more comprehensive studies that include other regions of the country are strongly recommended.

Acknowledgment. The authors gratefully acknowledge the Central Blood Bank Al Madinah Al Munawarah, KSA, for providing blood samples. We would also like to thank Cambridge Proofreading, LLC, for the English language editing.

\section{References}

1. Kim H, Webster RG, Webby RJ. Influenza virus: dealing with a drifting and shifting pathogen. Viral Immunol 2018; 31: 174-183.

2. Ryan EL, Hollingworth R, Grand RJ. Activation of the DNA damage response by RNA viruses. Biomolecules 2016; 6: 2 .

3. Yang G, Hodges EN, Winter J, Zanders N, Shcherbik S, Bousse T, et al. Development of an RNA strand-specific hybridization assay to differentiate replicating versus non-replicating influenza A virus. Journal of clinical microbiology 2020.

4. Iuliano AD, Roguski KM, Chang HH, Muscatello DJ, Palekar $\mathrm{R}$, Tempia S, et al. Estimates of global seasonal influenzaassociated respiratory mortality: a modelling study. Lancet 2018; 391: 1285-1300.

5. Lee VJ, Ho ZJM, Goh EH, Campbell H, Cohen C, Cozza $\mathrm{V}$, et al. Advances in measuring influenza burden of disease. Influenza Other Respir Viruses 2018; 12: 3-9.

6. Krammer F, Smith GJD, Fouchier RAM, Peiris M, Kedzierska K, Doherty PC, et al. Influenza. Nat Rev Dis Primers 2018; 4: 3.

7. Paget J, Spreeuwenberg P, Charu V, Taylor RJ, Iuliano AD, Bresee J, et al. Global mortality associated with seasonal influenza epidemics: new burden estimates and predictors from the GLaMOR Project. J Glob Health 2019; 9: 020421.
8. Sellers SA, Hagan RS, Hayden FG, Fischer WA 2nd. The hidden burden of influenza: a review of the extra-pulmonary complications of influenza infection. Influenza Other Respir Viruses 2017; 11: 372-393.

9. Hirve S, Lambach P, Paget J, Vandemaele K, Fitzner J, Zhang W. Seasonal influenza vaccine policy, use and effectiveness in the tropics and subtropics - a systematic literature review. Influenza Other Respir Viruses 2016; 10: 254-267.

10. Mahallawi WH. Assessment of the influenza vaccine (VAXIGRIP) in triggering a humoural immune response. $J$ Taibah Univ Med Sci 2017; 12: 523-527.

11. Chen YQ, Wohlbold TJ, Zheng NY, Huang M, Huang Y, Neu $\mathrm{KE}$, et al. Influenza infection in humans induces broadly crossreactive and protective neuraminidase-reactive antibodies. Cell 2018; 173: 417-429.

12. Krammer F. The human antibody response to influenza $A$ virus infection and vaccination. Nat Rev Immunol 2019; 19: 383-397.

13. McVernon J, Laurie K, Faddy H, Irving D, Nolan T, Barr I, et al. Seroprevalence of antibody to influenza A (H1N1)pdm09 attributed to vaccination or infection, before and after the second (2010) pandemic wave in Australia. Influenza Other Respir Viruses 2014; 8: 194-200.

14. Krumbholz A, Lange J, Dürrwald R, Walther M, Müller TH, Kühnel D, et al. Prevalence of antibodies to European porcine influenza viruses in humans living in high pig density areas of Germany. Med Microbiol Immunol 2014; 203: 13-24.

15. Andersen CJ, Murphy KE, Fernandez ML. Impact of obesity and metabolic syndrome on immunity. Adv Nutr 2016; 7 : 66-75.

16. Honce R, Schultz-Cherry S. Impact of obesity on Influenza A virus pathogenesis, immune response, and evolution. Front Immunol 2019; 10: 1071.

17. Green WD, Beck MA. Obesity impairs the adaptive immune response to influenza virus. Ann Am Thorac Soc 2017; 14: S406-S409.

18. Alomari MA, Khabour OF, Alzoubi KH. Metabolic differences between men and women who are long-term users of the water pipe: The Irbid WiHi project. J Vasc Nurs 2020; 38: 18-24.

19. Alsuwaidi AR, Al-Mekaini LA, Kamal SM, Narchi H, Souid AK. Seroprevalence of influenza A and B viruses among unvaccinated children in the United Arab Emirates: a crosssectional study. BMC Res Notes 2017; 10: 379.

20. Caini S, El-Guerche Séblain C, Ciblak MA, Paget J. Epidemiology of seasonal influenza in the Middle East and North Africa regions, 2010-2016: circulating influenza A and B viruses and spatial timing of epidemics. Influenza Other Respir Viruses 2018; 12: 344-352.

21. Abdalla O, Mohammed M, Hakawi AM, Aljifri A, Abdalla M, Eltigani S, et al. Hospital-based surveillance of influenza A (H1N1) pdm09 virus in Saudi Arabia, 2010-2016. Ann Saudi Med 2020; 40: 1-6.

22. Alrashid M, Taleb AA, Hajeer A, Arabi Y. Prevalence of antibodies against the Middle East respiratory syndrome coronavirus, influenza A and B viruses among blood donors, Saudi Arabia. Ann Thorac Med 2017; 12: 217-218.

23. Su S, Chaves SS, Perez A, D’Mello T, Kirley PD, Yousey-Hindes $\mathrm{K}$, et al. Comparing clinical characteristics between hospitalized adults with laboratory-confirmed influenza $\mathrm{A}$ and $\mathrm{B}$ virus infection. Clin Infect Dis 2014; 59: 252-255. 
24. Muñoz IS, Rello SR, Lejarazu RO. [Prevalence of antibodies and humoral response after seasonal trivalent vaccination against influenza B lineages in an elderly population of Spain]. Enferm Infecc Microbiol Clin 2018; 36: 572-575. [In Spanish]

25. Gaglani M, Vasudevan A, Raiyani C, Murthy K, Chen W, Reis $\mathrm{M}$, et al. Effectiveness of trivalent and quadrivalent inactivated vaccines against influenza B in the United States, 2011-2012 to 2016-2017. Clin Infect Dis 2020: ciaa102.

26. Vijaykrishna D, Holmes EC, Joseph U, Fourment M, Su YC, Halpin R, et al. The contrasting phylodynamics of human influenza B viruses. Elife 2015; 4: e05055.

27. Skowronski DM, Chambers C, De Serres G, Sabaiduc S, Winter AL, Dickinson JA, et al. Age-related differences in influenza B infection by lineage in a community-based sentinel system, 2010-2011 to 2015-2016, Canada. J Infect Dis 2017; 216: 697-702.

28. Xie H, Wan XF, Ye Z, Plant EP, Zhao Y, Xu Y, et al. H3N2 mismatch of 2014-15 northern hemisphere influenza vaccines and head-to-head comparison between human and ferret antisera derived antigenic maps. Sci Rep 2015; 5: 15279.

29. Hensley SE. Challenges of selecting seasonal influenza vaccine strains for humans with diverse pre-exposure histories. Curr Opin Virol 2014; 8: 85-89.

30. Rabaan AA, Alshaikh SA, Bazzi AM. Influenza A (H1N1) pdm09 epidemiology in the Eastern Province of Saudi Arabia. $J$ Infect Public Health 2018; 11: 636-639.
31. Van Kerkhove MD, Hirve S, Koukounari A, Mounts AW. Estimating age-specific cumulative incidence for the 2009 influenza pandemic: a meta-analysis of $\mathrm{A}(\mathrm{H} 1 \mathrm{~N} 1) \mathrm{pdm} 09$ serological studies from 19 countries. Influenza Other Respir Viruses 2013; 7: 872-886.

32. Dobner J, Kaser S. Body mass index and the risk of infection - from underweight to obesity. Clin Microbiol Infect 2018; 24: 24-28.

33. Alfelali M, Barasheed O, Tashani M, Azeem MI, El Bashir H, Memish ZA, et al. Changes in the prevalence of influenza-like illness and influenza vaccine uptake among Hajj pilgrims: a 10-year retrospective analysis of data. Vaccine 2015; 33 : 2562-2569.

34. Al-Tawfiq JA, Benkouiten S, Memish ZA. A systematic review of emerging respiratory viruses at the Hajj and possible coinfection with streptococcus pneumoniae. Travel Med Infect Dis 2018; 23: 6-13.

35. Benkouiten S, Charrel R, Belhouchat K, Drali T, Nougairede A, Salez N, et al. Respiratory viruses and bacteria among pilgrims during the 2013 Hajj. Emerg Infect Dis 2014; 20: 1821-1827.

36. Benkouiten S, Gautret P, Belhouchat K, Drali T, Nougairede A, Salez N, et al. Comparison of nasal swabs with throat swabs for the detection of respiratory viruses by real-time reverse transcriptase PCR in adult Hajj pilgrims. J Infect 2015; 70: 207-210. 